\title{
A Case of Peritoneal Dissemination and Splenic Metastasis after Gastric Cancer Surgery That Could Be Controlled with Multidisciplinary Treatment
}

\author{
Nozomi Karakuchi ${ }^{a}$ Senichiro Yanagawa ${ }^{a}$ Daisuke Takei ${ }^{a}$ \\ Shinya Kodama ${ }^{a}$ Yukio Takeshima ${ }^{b}$ Kazuo Sumimoto ${ }^{a, c}$ \\ aDepartment of Surgery, Yoshida General Hospital, Akitakata City, Japan; ${ }^{b}$ Department \\ of Pathology, Graduate School of Biomedical and Health Sciences, Hiroshima University, \\ Hiroshima, Japan; 'Department of Gastroenterological and Transplant Surgery, Graduate \\ School of Biomedical and Health Sciences, Hiroshima University, Hiroshima, Japan
}

\section{Keywords}

Gastric cancer · Splenic metastasis · Peritoneal dissemination · Multidisciplinary treatment

\begin{abstract}
Splenic metastasis is known to occur at the terminal stage of cancer. While peritoneal dissemination is the most frequent recurrence of gastric cancer, metastasis to the spleen from gastric cancer is very rare. Splenectomy is currently the most effective probable treatment for solitary splenic metastasis of gastric cancer; it provides a good patient prognosis. However, careful consideration of surgical indications is required, as splenic metastasis is typically accompanied by multi-organ metastasis and dissemination. One of the main cancer treatment methods is the "wait-and-see" approach using chemotherapy. In general, patients with gastric cancer are treated with systemic chemotherapy for distant metastases including peritoneal dissemination. Radiotherapy is not commonly used to prolong life in patients with gastric cancer as gastric cancer is predominantly adenocarcinoma. However, a recent report indicated that chemo-radiotherapy was performed successfully for unresectable gastric cancer, including peritoneal dissemination. Here, we present the case of a 67-year-old patient who was observed to have peritoneal dissemination and splenic metastasis after gastric cancer surgery. Once the peritoneal dissemination was localized, surgical excision and chemo-radiotherapy were performed. We treated the splenic metastasis with the "wait-and-see" strategy with chemotherapy. A complete response on imaging in accordance with the Response Evaluation Criteria in Solid Tumors was achieved using multidisciplinary treatment. Our strategy of intensive multidisciplinary therapy could be a treatment option for cases with peritoneal dissemination or splenic metastasis of gastric cancer.




\section{Introduction}

The spleen is the second largest solid organ of the reticuloendothelial system with a large number of monocytes that perform immunoglobulin synthesis production. Metastasis to the spleen is considered a rare event in malignant solid tumors and is part of multivisceral disease. Splenic metastasis is known to occur at the terminal stage of cancer, because it is usually accompanied by multiorgan metastasis and dissemination $[1,2]$. The primary tumors that metastasize to the spleen originate from the breast, lung, ovary, colorectal, or skin [2], with splenic metastasis of gastric cancer being less common. There are some reports where prognosis for solitary splenic metastasis treated with splenectomy is good $[3,4]$.

While splenic metastasis is very rare, peritoneal dissemination is the most frequent recurrence of gastric cancer. However, the mechanisms underlying peritoneal dissemination of gastric cancer have not been elucidated [5]. Recently, the therapeutic effect of intraperitoneal chemotherapy for peritoneal dissemination has been investigated [6], but it is not the current established treatment.

In general, patients with gastric cancer are treated with systemic chemotherapy for distant metastases, including peritoneal dissemination. Gastric cancer is predominantly an adenocarcinoma and radiation therapy is not commonly used as treatment; however, radiation therapy is one of the treatment pillars for general cancer management, regardless of the stage [7].

Here, we present a case of peritoneal dissemination and splenic metastasis that were observed after gastric cancer surgery. Complete response (CR) on the images was obtained in accordance with the Response Evaluation Criteria in Solid Tumors (RECIST) version 1.1 with multidisciplinary treatment including surgery, chemotherapy, and chemo-radiation therapy for recurrence and metastasis of gastric cancer.

\section{Case Report}

A 67-year-old man was diagnosed with gastric cancer located in the $U$ and M portion of the stomach. He underwent total gastrectomy and Roux-en-Y reconstruction with D2 lymphadenectomy for gastric cancer. The pathological examination showed a moderately differentiated adenocarcinoma in hematoxylin and eosin stain (H\&E) that was diagnosed as pT4aN2M0 pStage IIIB in accordance with the Union for International Cancer Control (UICC) tumor node metastasis (TNM) classification for gastric cancer.

He received oral administration of $\mathrm{S}-1\left(80 \mathrm{mg} / \mathrm{m}^{2}\right)$ for 14 consecutive days followed by 7 days of rest, every 3 weeks, as adjuvant chemotherapy. One year after gastric surgery, computed tomography (CT) imaging showed new nodules in the bladder rectal fossa and sigmoid mesocolon (Fig. 1a, b). We diagnosed these lesions as peritoneal dissemination of gastric cancer. He had no renal dysfunction; therefore, he was treated with $\mathrm{S}-1\left(80 \mathrm{mg} / \mathrm{m}^{2}\right)$ with cisplatin $\left(60 \mathrm{mg} / \mathrm{m}^{2}\right)$ referring to the SPIRITS trial [8]. After three cycles of S-1 with cisplatin, no new tumor lesions were found on $\mathrm{CT}$, but the total diameter of the two peritoneal disseminations increased by 38\% (Fig. 1c, d); therefore, we judged this as progressive disease in accordance with the RECIST version 1.1.

The chemotherapy treatment was changed to paclitaxel $\left(80 \mathrm{mg} / \mathrm{m}^{2}\right)$ with ramucirumab $(8$ $\mathrm{mg} / \mathrm{kg}$ ) based on the RAINBOW trial [9]. After six cycles of paclitaxel with ramucirumab, $\mathrm{SUV}_{\max }$ of ${ }^{18}$ F-FDG was increased to 8.9 in rectovesical excavation and 10.3 in sigmoid mesocolon (Fig. 2a, b), with no new accumulated lesion on positron emission tomography CT (PET-CT).

We then performed a trial laparotomy and observed the abdominal cavity. There were still only two peritoneal disseminated nodules, as shown in CT imaging, and peritoneal lavage

\section{Karger'}



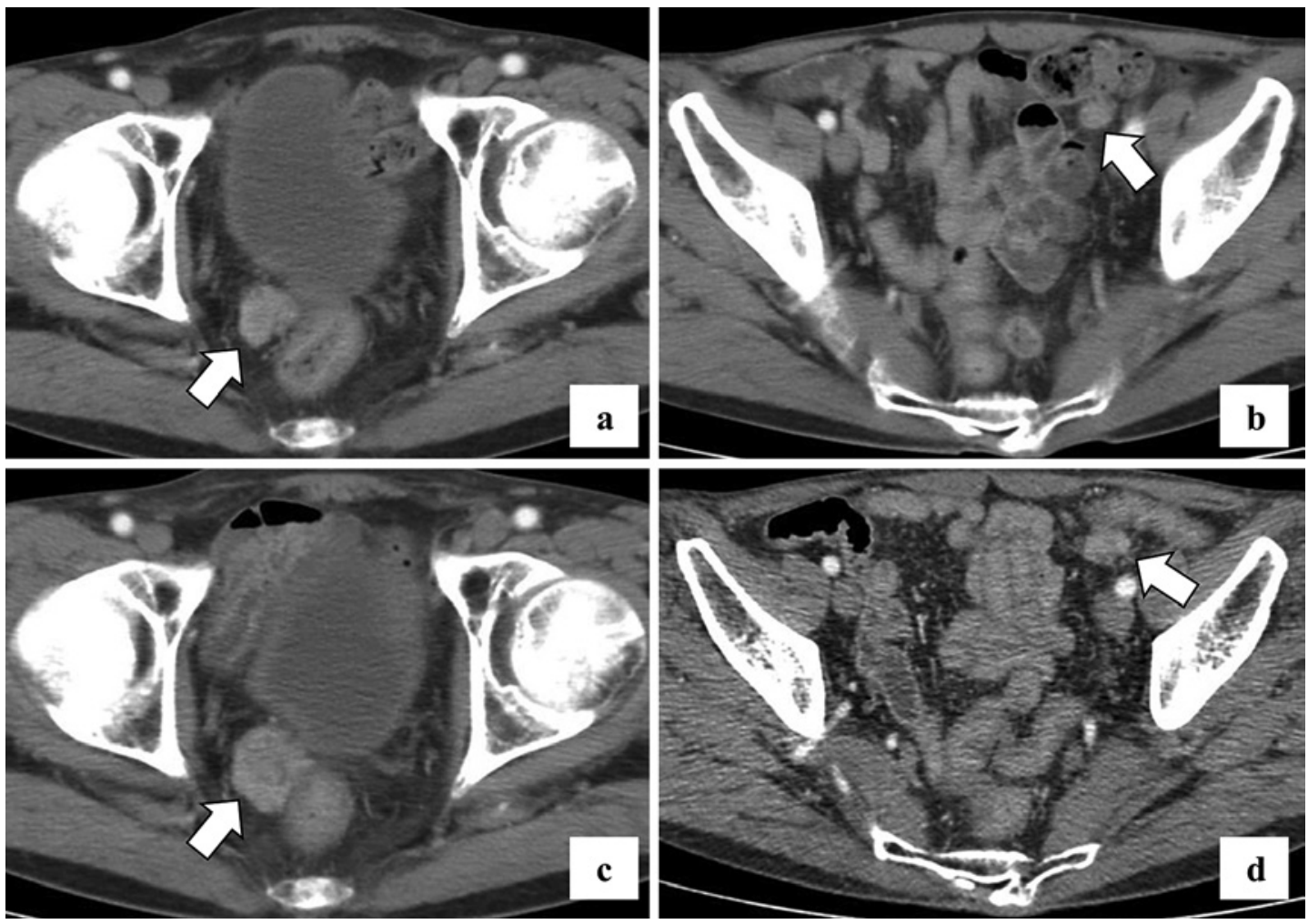

Fig. 1. CT scan with contrast showed nodules in rectovesical excavation (a) and sigmoid mesocolon (b) 1 year after gastric cancer surgery. After three cycle of S-1 with cisplatin, the diameter of both tumors in rectovesical excavation (c) and sigmoid mesocolon (d) increased.

cytology was negative. We partially resected the sigmoid colon, including the disseminated nodule in the mesocolon, but we could not perform a surgical resection of the disseminated nodule close to the bladder in the rectovesical excavation. The histological typing of the resected nodule of the mesocolon was the same as that of gastric cancer, and necrosis due to chemotherapy was observed in approximately $20 \%$ of the tumor. Therefore, we administered chemo-radiotherapy consisting of $\mathrm{S}-1\left(50 \mathrm{mg} / \mathrm{m}^{2}\right)$ and intensity-modulated radiation therapy with 66.0 Gy in 22 fractions for disseminated nodule in the rectovesical excavation.

Twelve months after chemo-radiotherapy, the $\mathrm{SUV}_{\max }$ of ${ }^{18} \mathrm{~F}-\mathrm{FDG}$ was reduced to $2.7 \mathrm{in}$ rectovesical excavation, but a new mass lesion $\left(\mathrm{SUV}_{\text {max }}: 9.4\right)$ appeared in the spleen on PET-CT (Fig. 2c). We judged that the accumulation of ${ }^{18} \mathrm{~F}$-FDG in the rectovesical excavation was an inflammatory change after chemo-radiation and diagnosed metachronous splenic metastasis of gastric cancer. Splenic metastasis is known as the terminal image of cancer and since there was a history of peritoneal dissemination, we introduced chemotherapy of capecitabine $\left(2,000 \mathrm{mg} / \mathrm{m}^{2}\right)$ with oxaliplatin $\left(130 \mathrm{mg} / \mathrm{m}^{2}\right)$ [9].

After ten cycles of capecitabine with oxaliplatin, there was no increase in $\mathrm{SUV}_{\max }$ of ${ }^{18} \mathrm{~F}-\mathrm{FDG}$ in rectovesical excavation and no ${ }^{18} \mathrm{~F}-\mathrm{FDG}$ uptake in the spleen on PET-CT (Fig. 2d). Therefore, we judged that it was a CR to treatment in accordance with RECIST version 1.1. Based on the observed side effect of grade 3 peripheral neuropathy due to the accumulation of oxaliplatin (Common Terminology Criteria for Adverse Events, version 5.0), chemotherapy was discontinued.

There has been no recurrence or metastasis on imaging for 15 months after the chemotherapy was concluded. 

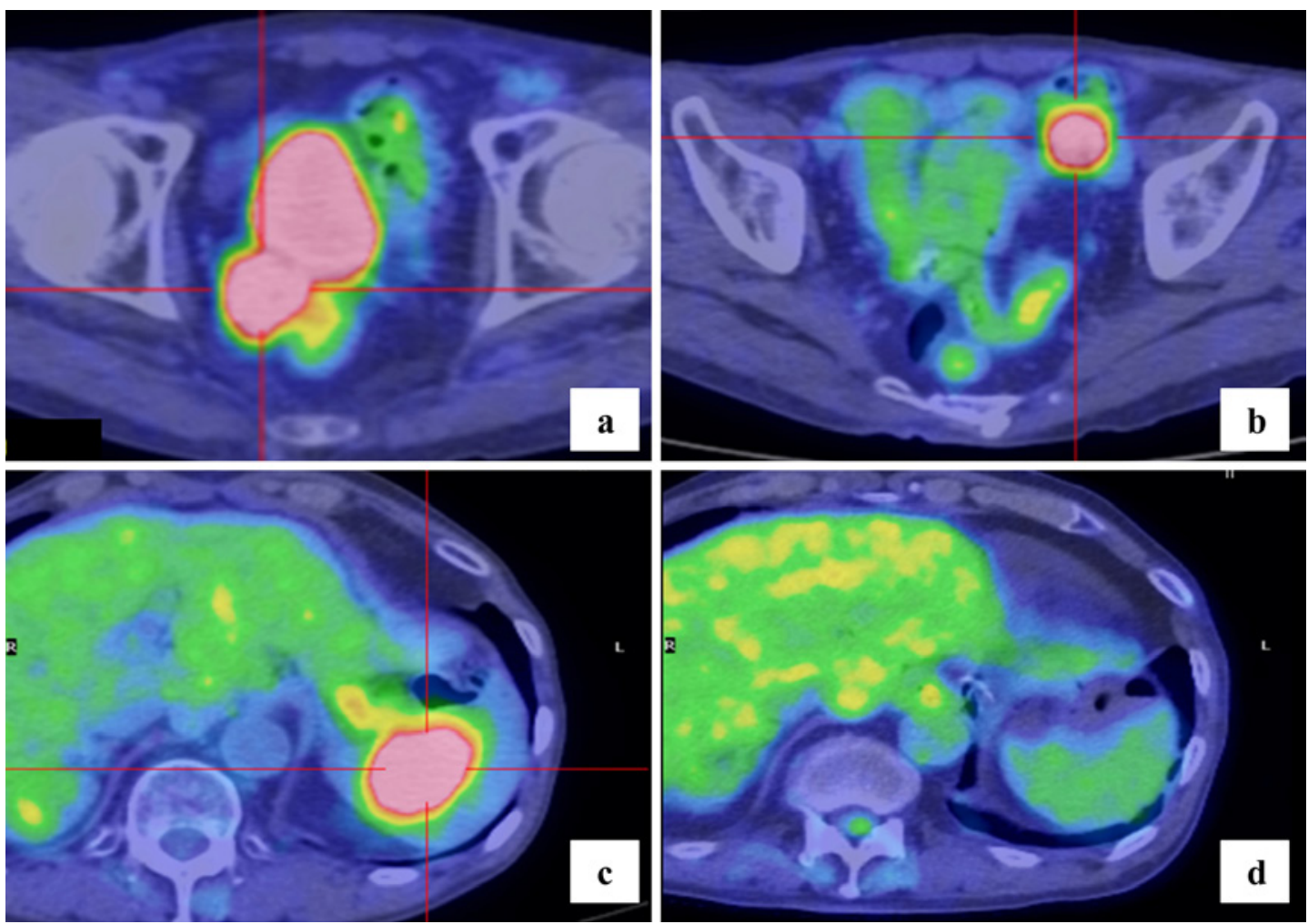

Fig. 2. After six cycles of paclitaxel with ramucirumab, $\mathrm{SUV}_{\max }$ of ${ }^{18} \mathrm{~F}-\mathrm{FDG}$ was 8.9 in rectovesical excavation (a) and 10.3 in sigmoid mesocolon (b) on PET-CT. Twelve months after chemo-radiotherapy was completed, PET-CT showed a new metastatic lesion with an $\mathrm{SUV}_{\max }$ of 9.4 that appeared in the spleen (c). After ten cycles of capecitabine with oxaliplatin, there was no ${ }^{18} \mathrm{~F}$-FDG uptake in the spleen (d).

\section{Discussion and Conclusion}

The rarity of splenic metastasis from solid malignant tumors has not been explained. The primary organs of origin for splenic metastasis are the breast, lung, colorectal, kidney, and ovarian carcinomas [2]. Metastases to the spleen from gastric cancer are very rare, regardless of their proximity anatomically.

As splenic metastases from gastric cancer are rarely documented, it continues to be difficult to predict the clinical behavior of this disease. Once splenic metastasis is clinically diagnosed, the patients' prognosis is poor since splenic metastasis is typically accompanied by multiorgan metastasis and dissemination $[1,2]$. Currently, the clinical diagnosis of splenic metastasis is largely dependent on a previous history of malignant disease. It is difficult to diagnose whether a splenic mass lesion is a primary tumor, metastatic tumor, vascular tumor, or infectious disorder [3]. Cavanna et al. [10] reported that splenic biopsy was an effective, safe, and inexpensive technique to obtain a definitive pathological diagnosis of a splenic mass, but it is generally avoided due to the risk of bleeding from the spleen or intra-abdominal dissemination of the tumor. On the other hand, a PET-CT scan is useful for differentiating between malignant and benign tumors, and for assessing metastasis to many organs or lymph nodes $[11,12]$. While splenectomy may be the effective and curative treatment of solitary splenic metastasis of gastric cancer [3,4], a splenic lesion is typically accompanied by multiple metastases at various other sites [1]. Therefore, a splenic metastatic lesion which is supposed to be "isolated" may represent an initial clinical manifestation of systemic metastases at 


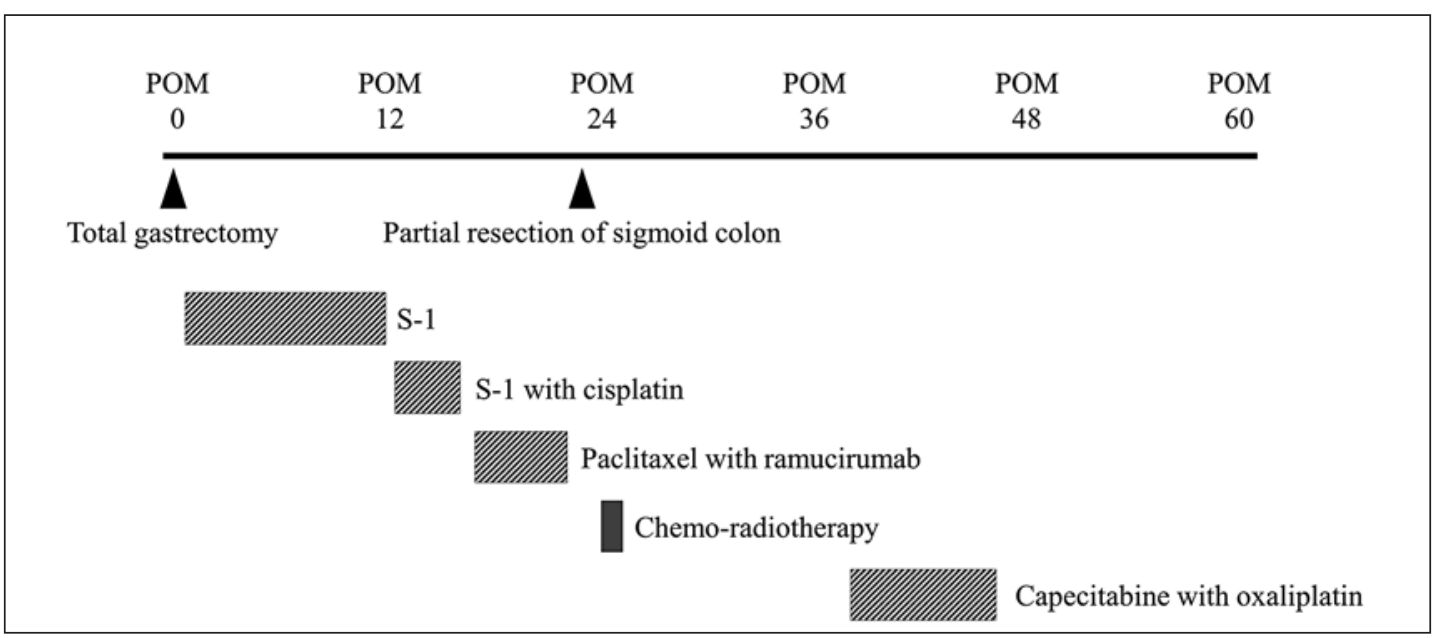

Fig. 3. Timeline of the clinical course of treatment including surgery, chemotherapy, and chemo-radiotherapy in postoperative months (POM). Total gastrectomy was performed in POM 0.

multiple sites [3]. In this case, if a splenectomy was performed, the patient would subsequently face the possibility of relapse in another organ. Therefore, the "wait-and-see" approach by chemotherapy has been defended as a way of selecting patients that truly benefit from aggressive surgical treatment $[3,13]$ and it is an effective way to understand the patient's general condition and cancer progression.

A case of localized peritoneal dissemination of gastric cancer is rare and its prognosis is poor. Chemotherapy is generally introduced, and intraperitoneal administration of paclitaxel has also been explored [6]. However, the therapeutic result was no better than expected, and an effective treatment method has not yet been established.

Radiotherapy is not commonly used to prolong life in patients with gastric cancer [7] because adenocarcinomas, such as gastric cancer, have a low sensitivity to radiotherapy. However, radiotherapy has highly effective local and regional effects for cancer treatment, such as relieving pain and other negative symptoms due to cancer invasion and bone metastasis [14]. In addition, recently a radical treatment combining chemotherapy with radiotherapy has been performed for unresectable gastric cancer [7, 15]. Locoregional radiation plus systemic chemotherapy may be effective for controlling a recurrence of gastric cancer and in the treatment of localized lesions with no distant metastases [7]. An accurate identification of the lesions involved, including peritoneal dissemination and the setting of the radiation field, is important to avoid recurrence after chemo-radiotherapy.

In this case, surgical treatment and chemo-radiotherapy were performed for localized peritoneal dissemination in the rectovesical excavation and mesocolon (Fig. 3). Additionally, since there was a history of peritoneal dissemination, and a possibility of distant metastases, we decided to perform a "wait-and-see" approach using chemotherapy instead of surgical resection for splenic metastasis. The chemotherapy of capecitabine with oxaliplatin was effective and CR on imaging was obtained according to RECIST version 1.1. Using this treatment, we could avoid the possibility of overwhelming post-splenectomy infection, where mortality is very high. There has been no recurrence or metastasis on imaging for 15 months after chemotherapy was finished.

Since the patient is still recurrence-free and alive 5 years after gastric surgery, our treatment strategy could be a treatment option for cases with localized peritoneal dissemination or spleen-only metastasis. 


\section{Case Reports in Oncology}

\begin{tabular}{l|l}
\hline Case Rep Oncol 2020;13:1164-1170 \\
\hline DOI: 10.1159/000510406 & $\begin{array}{l}\text { @ 2020 The Author(s). Published by S. Karger AG, Basel } \\
\text { www.karger.com/cro }\end{array}$ \\
\hline
\end{tabular}

Karakuchi et al.: Metastases of Gastric Cancer Treated with Multidisciplinary Treatment

\section{Acknowledgement}

The authors would like to thank the patient for providing consent to publish clinical information and data. We would like to thank Editage (www.editage.com) for English language editing.

\section{Statement of Ethics}

Written informed consent was obtained from the patient.

\section{Conflict of Interest Statement}

The authors have no conflicts of interest to declare.

\section{Funding Sources}

The authors received no financial support for the research, authorship, or publication of this article.

\section{Author Contributions}

N.K. and S.Y. made a substantial contribution toward the concept and design of the study and in data acquisition and interpretation. D.T., S.K., Y.T., and K.S. were involved in drafting the manuscript and critical revision of the intellectual content. S.Y. approved the final version of the manuscript submitted for publication. All authors read and approved the manuscript.

\section{References}

1 Schön CA, Görg C, Ramaswamy A, Barth PJ. Splenic metastases in a large unselected autopsy series. Pathol Res Pract. 2006 May;202(5):351-6.

2 Compérat E, Bardier-Dupas A, Camparo P, Capron F, Charlotte F. Splenic metastases: clinicopathologic presentation, differential diagnosis, and pathogenesis. Arch Pathol Lab Med. 2007 Jun;131(6):965-9.

3 Zhu YP, Mou YP, Ni JJ, Zhou YC, Jiang JW, Jiang ZN, et al. Isolated splenic metastases from gastric carcinoma: a case report and literature review. World J Gastroenterol. 2013 Aug;19(31):5199-203.

4 Yoshizawa J, Kubo N, Ishizone S, Karasawa F, Nakayama A. Curative resection by splenectomy for solitary splenic metastasis from early gastric cancer: a case report and literature review. BMC Cancer. 2017 Jan;17(1): 436.

5 Sun F, Feng M, Guan W. Mechanisms of peritoneal dissemination in gastric cancer. Onco Lett. 2017 Jun;14(6): 6991-8.

6 Ishigami H, Fujiwara Y, Fukushima R, Nashimoto A, Yabusaki H, Imano M, et al. Phase III trial comparing intraperitoneal and intravenous paclitaxel plus S-1 versus cisplatin plus S-1 in patients with gastric cancer with peritoneal metastasis: PHOENIX-GC trial. J Clin Oncol. 2018 Jul;36(19):1922-9.

7 Koizumi W, Narahara H, Hara T, Takagane A, Akiya T, Takagi M, et al. S-1 plus cisplatin versus S-1 alone for first-line treatment of advanced gastric cancer (SPIRITS trial): a phase III trial. Lancet Oncol. 2008 Mar; 9(3): 215-21.

8 Wilke H, Muro K, Van Cutsem E, Oh SC, Bodoky G, Shimada Y, et al. Ramucirumab plus paclitaxel versus placebo plus paclitaxel in patients with previously treated advanced gastric or gastro-oesophageal junction adenocarcinoma (RAINBOW): a double-blind, randomised phase 3 trial. Lancet Oncol. 2014 Nov;15(11):1224-35.

9 Ajani JA, D'Amico, TA, Almhanna K, Bentrem, DJ, J Chao, Das P, et al. Gastric Cancer, Version 3.2016, NCCN Clinical Practice Guidelines in Oncology. J Natl Compr Canc Netw. 2016 Oct;14(10):1286-312.

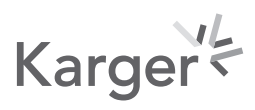


10 Cavanna L, Lazzaro A, Vallisa D, Civardi G, Artioli F. Role of image-guided fine-needle aspiration biopsy in the management of patients with splenic metastasis. World J Surg Oncol. 2007 Feb;5:13.

11 Metser U, Miller E, Kessler A, Lerman H, Lievshitz G, Oren R, et al. Solid splenic masses: evaluation with 18F-FDG PET/CT. J Nucl Med. 2005 Jan;46(1):52-9.

12 Kawasaki H, Kitayama J, Ishigami H, Hidemura A, Kaisaki S, Nagawa H. Solitary splenic metastasis from early gastric cancer: report of a case. Surg Today. 2010 Jan;40(1):60-3.

13 Taki T, Hoya Y, Watanabe A, Nakayoshi T, Okamoto T, Sekine H, et al. Usefulness of chemoradiotherapy for inoperable gastric cancer. Ann R Coll Surg Engl. 2017 Apr;99(4):332-6.

14 Shigeoka H, Imamoto H, Nishimura Y, Shimono T, Furukawa H, Imamura H, et al. Complete response to preoperative chemoradiotherapy in highly advanced gastric adenocarcinoma. World J Gastrointest Oncol. 2010 Jun; 2(6):282-6.

15 F Ren, S Li, Y Zhang, Z Zhao, H Wang, Y Cui, et al. Efficacy and safety of intensity-modulated radiation therapy versus three-dimensional conformal radiation treatment for patients with gastric cancer: a systematic review and meta-analysis. Radiat Oncol. 2019 May;14(1):84. 